\title{
Contributing Reviewers in 2016
}

J Pediatr Genet 2017;6:i-ii.

Journal of Pediatric Genetics wishes to recognize those who contributed as an expert peer reviewer of submitted scientific papers in 2016.

Thank you for your contributions to Journal of Pediatric Genetics in 2016.

Margaret Adam
Patrizia Agretti
Mehmet Arif Akşit
Joseph Alaimo
S. V. Andreieva
Maarten Arends
Christian Beetz
Zahurul Anthony Bhuiyan
Weimin Bi
Maria Paz Bidondo
Lynne Marie Bird
ML Bonora
Erwin Brosens
Bert Callewaert
Hakan Cangul
Matteo Cassina
Nicolas Chassaing
Sanjiv Choudhary
Paul de-sa-Júnior
Stephen I. Deutsch
Avinash Vijay Dharmadhikari
Sofia Douzgou
Darius Ebrahimi-Fakhari
Hala T. El-Bassyouni
Sara Elrefai
Ellouz Emna
Robert P. Ericson
Edward Esplin
Paolo Fontana
Melanie Fradin
Valentina Gatta
Alper Gezdirici
William T. Gibson
Chen H.
Manis Hajana
Susan J. Hassed
Sarah Heron
Hans L. J. Hoeve

Engela Honey

Albandar Jasim

Joanna Kabat-Koperska

Fakhri Kallabi

Hiroshi Kanno

Toshitaka Kawarai

Yskert von Kodolitsch

Katrin Koehler

Didier Lacombe

Li Lan

Dawn Laney

Pedro Luiz Squilacci Leme

Anna Lewinska

Karina Lezirovitz

Thomas Liehr

Li Liu

Leonardi Luca

Lionel Van Maldergem

Tulin Mansur

Fernando Augusto de Lima Marson

Divya Matta

Meenakshi Mehrotra

Ute Moog

Takayuki Morisaki

Hatice Mutlu

Kasiani Myers

Sunghee Oh

Antonio Orlacchio

Giandomenico Palka

Ashley Parrott

Antonia Perez-Aytes

Wladimir Bocca Vieira de Rezende Pinto

Harvey B. Pollard

Giovanni Ponti

Lorraine Potocki

Heiko Reutter

David Richman

Horacio Rivera 
Alan Rogol

Marian Ros

Khaled Saad

Daniele Santi

Saikat Santra

Mrinmoy Sanyal

Ravi Sasan

Ingrid Schierz

Rodney J. Scott

Marie-Christine Seghaye

Igor V. Sharakhov

Laurie Smith

Eva-Lena Stattin

Elisabeth Steichen-Gersdorf

Michael Anthony Swanson

Nathalie Tabibzadeh

Toshihiro Tajima

Hua Tang

Natacha Teissier liehr Thomas

Clara L. Thompson

Dick Tibboel

Hidefumi Tonoki

Ali Topak

Barbara Tudek

Ana Fernández Urbón

Eric Vallender

Marcal Vilar

Alexander O. Vortmeyer

S. Watanabe

Allison Waters

Rachel Wevrick

Zhoubing $\mathrm{Xu}$

Toshiyuki Yamamoto

Ruxu Zhang

Weiqiang Zhang

Vincent Zimmer 